Philosophical Magazine B, 1998, Vol. 78, Nos. 5/6, 429-433

\title{
$A b$ initio calculation of forces and lattice relaxations in metallic alloys
}

\author{
T. Korhonen, N. Papanikolaou, R. Zeller and P. H. Dederichs \\ Institut für Festkörperforschung, Forschungszentrum Jülich, D-52425 Jülich, \\ Germany
}

\begin{abstract}
We present first-principles calculations of forces and lattice relaxations in bcc Fe. In particular, relaxations around $3 \mathrm{~d}, 4 \mathrm{~d}$ and $5 \mathrm{~d}$ transition metal impurities are calculated. The calculations are based on a full-potential Korringa-KohnRostoker Green's function method for defects and employ the local spin density approximation for the exchange and correlation effects. The nonspherical parts of the potential and the charge density are treated correctly, while the forces are calculated by an ionic version of the Hellmann-Feynman theorem. Lattice statics methods are used to describe the longer ranged relaxations. The results are compared with lattice parameter measurements for the volume changes. Because of the correct treatment of the sharp shape of the Wigner-Seitz cell, the angular momentum expansion coefficients of the cell potential have discontinuities in the first derivative, which cause some complications when solving the radial equations. An effective method to get around these discontinuities is introduced.
\end{abstract}

\section{$\S 1$. Introduction}

The KKR method of electronic structure calculations, introduced by Korringa (Korringa 1947) and Kohn and Rostoker (Kohn and Rostoker 1965), is well known for its mathematical rigour and elegance. By taking advantage of multiple scattering theory, the method clearly separates the scattering properties of the single potentials from the geometrical structure of the system. The Green's function (GF) version of the KKR method was introduced by Beeby and others (Dupree 1961, Beeby 1967, Holzwarth 1975) and has later on been used by the authors in extensive calculations for impurities in metals (Zeller and Dederichs 1979, Podloucky et al. 1980, Braspenning et al. 1984). The GF version is particularly useful for density-functional calculations.

In this paper we discuss some technical problems of our full-potential KKR-GF method connected with discontinuities of the shape functions which describe the form of the Wigner-Seitz (WS) cell and show how these problems can be solved efficiently by introducing 'smearing' procedures. We then calculate the lattice relaxations of transition metal impurities in $\mathrm{Fe}$ and compare these with experimental data.

\section{§2. Full-potential KKR-Green's function method}

In multiple-scattering theory, the whole space is divided into non-overlapping and space-filling cells around each atomic site $\mathbf{R}^{n}$. The crystal Green's function $G$ is then written in a cell-centred double expansion around the positions $\mathbf{R}^{n}$ and $\mathbf{R}^{n \text { in }}$ in 
different cells as (Podloucky et al. 1980)

$$
G\left(\mathbf{r}+\mathbf{R}^{n}, \mathbf{r}^{\prime}+\mathbf{R}^{n^{\prime}} ; E\right)=G_{\mathrm{s}}^{n}\left(\mathbf{r}, \mathbf{r}^{\prime} ; E\right) \delta_{n n^{\prime}}+\sum_{L, L^{\prime}} R_{L}^{n}(\mathbf{r} ; E) G_{L L}^{n n^{\prime}}(E) R_{L^{\prime \prime}}^{n^{\prime}}\left(\mathbf{r}^{\prime} ; E\right),
$$

where $G_{\mathrm{s}}^{n}\left(\mathbf{r}, \mathbf{r}^{\prime} ; E\right)$ represents the Green's function for a single potential confined inside the cell $n$ in otherwise free space. In the above equation, the position vectors $\mathbf{r}$ and $\mathbf{r}^{\prime}$ are restricted to the Wigner-Seitz cell and the index $L$ denotes the angular momentum quantum numbers $(l, m)$. The partial wave $R_{L}^{n}(\mathbf{r} ; E)$ is defined as the solution of the Lippmann-Schwinger equation for a spherical wave $j_{l}(r \sqrt{ } E) Y_{L}(\hat{\mathbf{r}})$ incident on the potential $V^{n}(\mathbf{r})$ at the site $\mathbf{R}^{n}$. In our full-potential KKR-GF method, the potentials and the partial waves are expanded in real spherical harmonics, $Y_{L}$, as $V^{n}(\mathbf{r})=\sum_{L} V_{L}^{n}(r) Y_{L}(\hat{\mathbf{r}})$ and $R_{L}^{n}(\mathbf{r} ; E)=\sum_{L}(1 / r) R_{L, L}^{n}(r ; E) Y_{L},(\hat{\mathbf{r}})$, respectively, and for the analytically known free electron Green's function $g\left(\mathbf{r}, \mathbf{r}^{\prime} ; E\right)$, is expanded in a double expansion as $g\left(\mathbf{r}, \mathbf{r}^{\prime} ; E\right)=\sum_{L}\left(1 / r r^{\prime}\right) Y_{L}(\hat{\mathbf{r}}) g_{l}\left(r, r^{\prime} ; E\right) Y_{L}\left(\hat{\mathbf{r}}^{\prime}\right)$. Using these expansions, one gets the following set of coupled radial equations for the above wave-functions $R_{L L}^{n},(r ; E)$

$$
R_{L L^{\prime}}^{n}(r ; E)=r j_{l}(r \sqrt{ } E) \delta_{L L^{\prime}}+\int \mathrm{d} r^{\prime} g_{l}\left(r, r^{\prime} ; E\right) \sum_{L^{\prime \prime}} V_{L^{\prime \prime}}^{n}\left(r^{\prime}\right) R_{L^{\prime \prime} L^{\prime}}^{n}\left(r^{\prime} ; E\right),
$$

where $j_{l}$ are the spherical Bessel functions and $V_{L L}^{n},(r)$ is the double indexed potential $V_{L^{\prime}}^{n}(r)=\sum_{L^{\prime \prime}} C_{L^{\prime} L^{\prime \prime}} V_{L^{\prime \prime}}(r)$ with Gaunt coefficients $C_{L^{\prime}, L^{\prime \prime}}$. The non-spherical part of the potential, $\Delta V^{n}(\mathbf{r})=V^{n}(\mathbf{r})-V_{0}^{n}(r)$, couples the angular momentum channels. These equations can be solved quite efficiently by the following procedure. First, calculate the radial solutions, $R_{l}^{0, n}(r ; E)$, and the radial Green's function, $G_{l}^{0, n}\left(r, r^{\prime} ; E\right)$, using only the spherical part of the potential, $V_{0}^{n}(r)$, and then calculate the full solutions, $R_{L L}^{n},(r ; E)$, iteratively by using an integral equation with $\Delta V^{n}(\mathbf{r})$ as a perturbative potential,

$$
R_{L L^{\prime}}^{n}\left(r^{\prime} ; E\right)=R_{l}^{0, n}(r ; E) \delta_{L L^{\prime}}+\int \mathrm{d} r^{\prime} G_{l}^{0, n}\left(r, r^{\prime} ; E\right) \sum_{L^{\prime \prime}} \Delta V_{L L^{\prime \prime}}^{n}\left(r^{\prime}\right) R_{L^{\prime \prime} L^{\prime}}^{n}\left(r^{\prime} ; E\right) .
$$

The corresponding Born series converges rapidly, and in most cases the second Born approximation, i.e., the second iterative step, is already sufficient for an accurate solution to the coupled radial equations (Drittler et al. 1991).

In the full-potential KKR method used here, the shape of the different cells is described by the shape functions $\Theta^{n}(\mathbf{r})$, being equal to unity inside the cell $n$ and vanishing everywhere else. These functions are also expanded in real spherical harmonics as

$$
\Theta^{n}(\mathbf{r})=\sum_{L} \Theta_{L}^{n}(r) Y_{L}(\hat{\mathbf{r}}) .
$$

While the $l$-convergence of the expansion (4) is rather slow, the overall convergence is determined by the $l$ cut-off of the wave functions $R_{L, L}^{n}$. If here angular momenta up to $l_{\max }$ are taken into account, then the expansion of the charge density and the potential includes only contributions up to $2 l_{\max }$, while the evaluation of the Coulomb and exchange-correlation terms in the potential energy requires $l$-terms up to $4 l_{\max }$. Thus only shape functions $\Theta_{L}^{n}(r)$ up to $4 l_{\max }$ are needed in the calculations. 
A problem connected with the use of shape functions is that the expansion coefficients $\Theta_{L}^{n}(r)$ have kinks, i.e. discontinuities of the first derivative, for radii that corresponds to the distance of a face, an edge, or a vertex of the polyhedron from the centre (Papanikolaou et al. 1997). Since a shape function truncates the potential within a WS cell, these discontinuities are also present in the first derivative of the potential. To avoid inaccuracies in the numerical integrations of the radial equations caused by the kinks, one could restart the integrations of the equations at each kink. This procedure works well for symmetric polyhedra like the WS cell of the fcc lattice, where one finds only 3 kinks, but this procedure is not very efficient for non-symmetric polyhedra with many kinks, since in the solution of the coupled radial equations many mesh points have to be used.

In this work the (possibly) distorted lattice is described by keeping the same space division as in the undistorted lattice, but the shape functions are expanded around the shifted atomic positions, so the shape functions (and potential) have many kinks, typically as many as 30 or 40 . In the present work, this problem is avoided by using a relatively sparse radial mesh and interpolating the shape functions to this mesh. To avoid the discontinuities, a smeared spherical potential, $\widetilde{V}_{0}^{n}(r)$, is used in the radial equation and the difference between the true spherical potential and the smeared one, $\Delta V_{0}^{n}(r)=V_{0}^{n}(r)-\widetilde{V}_{0}^{n}(r)$, is put to the Born series part of the solution of the coupled radial equations. Now the spherical radial problem can be solved without any complications arising from the kinks since these only enter in the integrand of the $r$ integration, so one can strongly reduce the number of radial mesh points needed. Another way is the method proposed by Papanikolaou et al. (1997), where the sharp Wigner-Seitz cell boundaries are smoothed by a Fermi function when calculating corresponding shape functions. The calculated forces are insensitive to the small approximations made in the shape of the WS cell, but some care should be taken in both schemes when calculating the total energy to assure, that it has the desired extremal properties. This is clearly seen from figure 1 where the total energy of $\mathrm{Cu}$ impurity in bcc iron is plotted as a function of the relaxation of the nearest neighbour (NN) iron atoms. Using smeared shapes everywhere does not give the correct form for the energy parabola around the $0 \%$ relaxation and has also a small overall shift to lower energies. This discrepancy arises from small errors made in the calculation of the Coulomb energy, $U[n]$, and the exchange correlation energy, $E_{x c}[n]$. In order to preserve the extremal properties, one has to calculate these partial energies correctly by using the exact shapes with kinks. If this is done, then the points fall back to the same line as the results calculated using the correct shapes with kinks. Thus both smoothing procedures work well and give reliable total energies and forces.

\section{$\S 3$. LATTICE DisPlaCEMENTS A ROUND IMPU RITIES IN BCC IRON}

We have performed calculations of lattice displacements around late $3 \mathrm{~d}, 4 \mathrm{~d}$ and $5 \mathrm{~d}$ transition metal impurities in bec Fe. The calculations were done using a cluster of 59 perturbed potentials, so that an impurity and the $5 \mathrm{NN}$ bcc shells around the impurity were treated self-consistently. The theoretical value for the lattice constant, $5.22 \mathrm{au}$, obtained by a total energy minimization is used in the calculations. In the calculations angular momenta up to $\ell_{\max }=4$ are included for the radial functions and Green's function and exchange correlation effects are treated using the LDA parametrization of Vosko et al. (1980). The energy integrations were done in the complex energy plane using a Fermi-Dirac distribution for the occupation function 


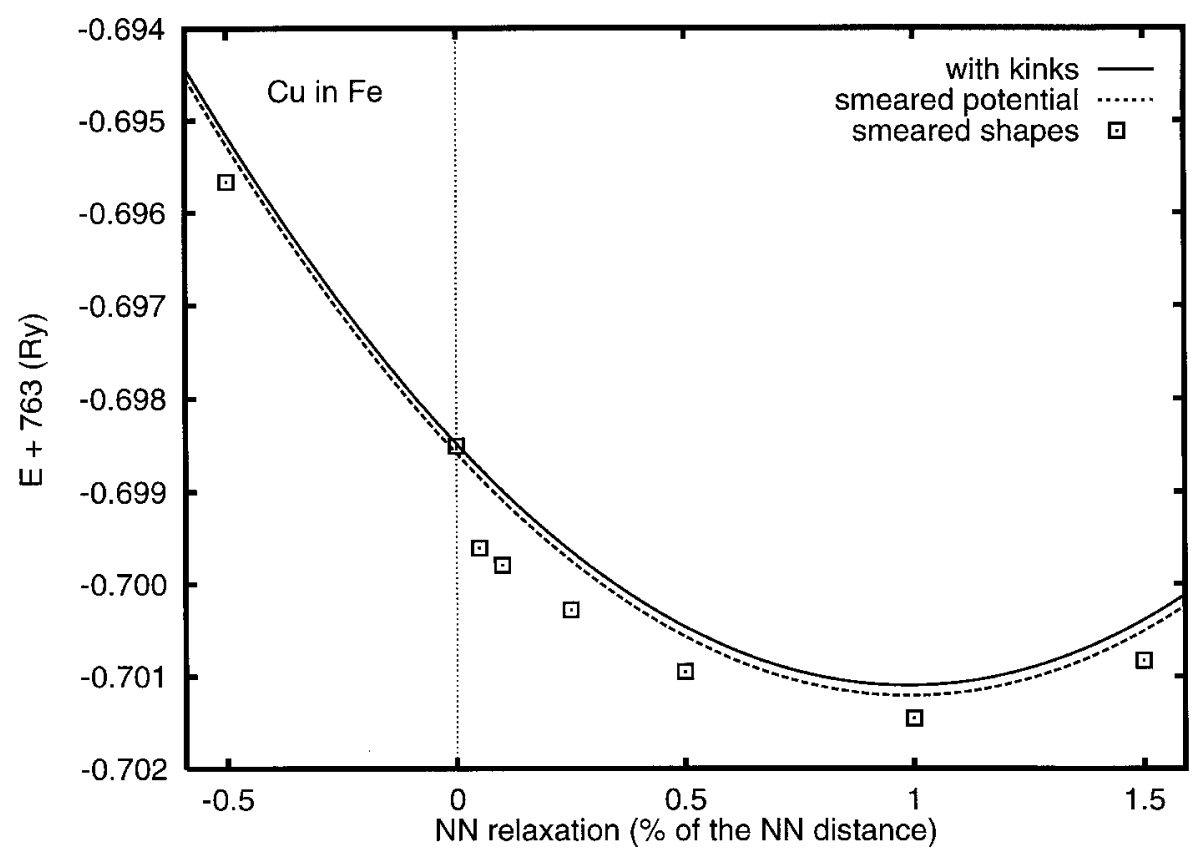

Figure 1. Change in the total energy for $\mathrm{Cu}$ impurity in bcc $\mathrm{Fe}$ as a function of the NN displacements. Shown are results of calculations using shapes with kinks (solid line), smeared shapes (open squares) and the now proposed method with $\Delta V_{0}^{n}$ in the Born approximation part (dashed line).

Table 1. The calculated first and second NN displacements ( $\%$ of the NN distance) for late $3 \mathrm{~d}, 4 \mathrm{~d}$, and $5 \mathrm{~d}$ transition metal impurities in bcc iron. Also shown are the estimated relative volume changes together with the experimental data from lattice-parameter measurements (King 1966).

\begin{tabular}{lrrrrrrrrr}
\hline & \multicolumn{1}{c}{$\mathrm{Co}$} & \multicolumn{1}{c}{$\mathrm{Ni}$} & $\mathrm{Cu}$ & \multicolumn{1}{c}{$\mathrm{Rh}$} & \multicolumn{1}{c}{$\mathrm{Pd}$} & $\mathrm{Ag}$ & $\mathrm{Ir}$ & $\mathrm{Pt}$ & $\mathrm{Au}$ \\
\hline NN rel $(\%)$ & 0.1 & 0.7 & 1.2 & 2.3 & 3.1 & 3.8 & 2.5 & 3.2 & 3.9 \\
NNN rel $(\%)$ & -0.0 & -1.1 & -1.2 & 0.8 & 0.2 & 0.0 & 0.8 & 0.8 & 0.3 \\
$\left(\Delta V / V_{0}\right)^{\text {theo }}(\%)$ & 4.1 & 3.9 & 11.3 & 37.6 & 43.1 & 54.4 & 40.9 & 50.6 & 56.2 \\
$\left(\Delta V / V_{0}\right)^{\exp }(\%)$ & 1.5 & 4.6 & 17.5 & & 62.2 & & & & 44.2 \\
\hline
\end{tabular}

(Wildberger et al. 1995). The results for first and second $\mathrm{NN}$ displacements together with the estimated macroscopic volume changes are summarized in the table 1 . The calculated volume changes, which are calculated using lattice-statics simulations based on the Kanzaki method (Leibfried and Breuer 1978), are in a good agreement with the experimental data from lattice-parameter measurements (King 1966).

\section{$\S 4$. Summa RY}

We have reported here $a b$ initio calculations of lattice relaxation effects around late $3 \mathrm{~d}, 4 \mathrm{~d}$ and $5 \mathrm{~d}$ transition metal impurities in bcc iron. The calculated macroscopic volume changes induced by the defect are in good agreement with the available lattice-parameter measurements. The calculations show that within the fullpotential KKR method accurate force and energy calculations are possible. The 
problems arising with the exact treatment of the WS cell, i.e. discontinuities of the first derivative in the angular momentum expansion of the cell potential, can be elimimated and the method retains its efficiency also for problems with non-symmetric WS cells. The described force and relaxation calculations can be used in connection with our recently developed version of the tight-binding KKR method (Wildberger et al. 1997, Zeller 1997).

\section{ACKNOWLEDGMENTS}

This work has been supported by a bilateral German-Greek cooperation grant and was partially funded by the TMR Network 'Ab initio calculations of magnetic properties of surfaces, interfaces and multilayers' (Contract: ERBFMRXCT960089).

Beeby, J. L., 1967, Prog. Roy. Soc. A, 302, 113.

\section{REFERENCES}

Braspenning, P. J., Zeller, R., Lodder, A., and Dederichs, P. H., 1984, Phys. Rev. B, 29, 703.

Drittler, B., Weinert, M., Zeller, R., and Dederichs, P. H., 1991, Solid State Commun., 79, 31 .

Dupree, Th. H., 1961, Ann. Phys., 15, 63.

Holzwarth, N. A. W., 1975, Phys. Rev. B, 11, 3718.

KING, H. W., 1966, J. Mater. Sci., 1, 79.

Kohn, W., and Rostoker, N., 1954, Phys. Rev., 94, 1111.

Korringa, J., 1947, Physica, 13, 392.

Leibfried, G., and Breuer, N., 1978, Point Defects in Metals I (Berlin: Springer-Verlag).

Lodder, A., 1976, J. Phys. F, 6, 1885.

Papanikolaou, N., Zeller, R., Dederichs, P. H., and Stefanou, N., 1997, Phys. Rev. B, 55, 4157.

Podloucky, R., Zeller, R., and Dederichs, P. H., 1980, Phys. Rev. B, 22, 5777.

Vosko, S. H., Wilk, L., and Nusair, M., 1980, J. Can. Phys., 58, 1200.

Wildberger, K., Lang, P., Zeller, R., and Dederichs, P. H., 1995, Phys. Rev. B, 52, 11502.

Wildberger, K., Zeller, R., and Dederichs, P. H., 1997, Phys. Rev. B, 55, 10074.

Zeller, R., 1997, Phys. Rev. B, 55, 9400.

Zeller, R., and Dederichs, P. H., 1979, Phys. Rev. Lett., 42, 1713. 